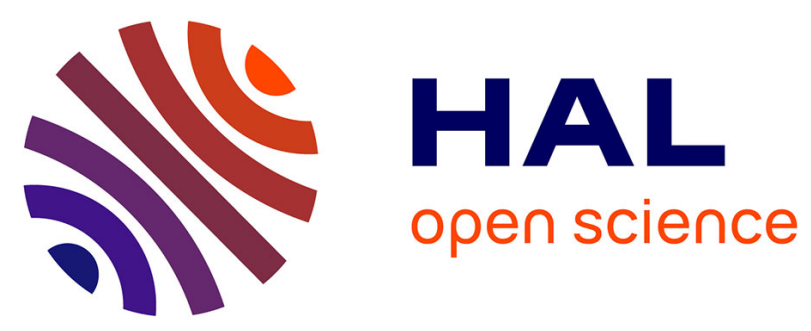

\title{
Does the T1 subdivision correlate with the risk of recurrence of papillary thyroid cancer?
}

Nathalie Chereau, Christophe Trésallet, Severine Noullet, Gaelle Godiris-Petit, Frédérique Tissier, Laurence Leenhardt, Fabrice Menegaux

\section{- To cite this version:}

Nathalie Chereau, Christophe Trésallet, Severine Noullet, Gaelle Godiris-Petit, Frédérique Tissier, et al.. Does the T1 subdivision correlate with the risk of recurrence of papillary thyroid cancer? Langenbeck's Archives of Surgery, 2016, 401 (2), pp.223-230. 10.1007/s00423-016-1399-y . hal01318374

\section{HAL Id: hal-01318374 \\ https://hal.sorbonne-universite.fr/hal-01318374}

Submitted on 19 May 2016

HAL is a multi-disciplinary open access archive for the deposit and dissemination of scientific research documents, whether they are published or not. The documents may come from teaching and research institutions in France or abroad, or from public or private research centers.
L'archive ouverte pluridisciplinaire HAL, est destinée au dépôt et à la diffusion de documents scientifiques de niveau recherche, publiés ou non, émanant des établissements d'enseignement et de recherche français ou étrangers, des laboratoires publics ou privés. 


\section{Does the T1 subdivision correlate with the risk of recurrence of papillary thyroid cancer?}

Nathalie Chereau, MD, ${ }^{\mathrm{a}}$ Christophe Trésallet, MD, PhD, ${ }^{\mathrm{a}}$ Severine Noullet, MD, Gaelle GodirisPetit, MD, Frédérique Tissier, $\mathrm{MD}, \mathrm{PhD},{ }^{\mathrm{b}}$ Laurence Leenhardt, $\mathrm{MD}, \mathrm{PhD},{ }^{\mathrm{c}}$ and Fabrice Menegaux, $\mathrm{MD}, \mathrm{PhD}^{\mathrm{a}}$

From the Departments of General and Endocrine Surgery, ${ }^{a}$ Pathology ${ }^{b}$ and Nuclear Medicine ${ }^{c}$ Hospital Pitié Salpêtrière, APHP, University Pierre and Marie Curie (Paris VI), Paris, France

Corresponding author: Fabrice Menegaux, MD, PhD

fabrice.menegaux@aphp.fr

Service de Chirurgie Générale, Hôpital de la Pitie Salpêtrière, 47-83 boulevard de l'hôpital, 75651

Paris Cedex 13, France. Tel.: +33 1421766 32; fax: +33 142176607.

The authors have no conflicts of interest to declare.

\section{Contribution}

Study conception and design:

Data acquisition:

Data analysis and interpretation:

Drafting of manuscript:

Critical revision of manuscript:

\section{Author(s)}

Chereau, Trésallet, Godiris Petit, Noullet, Tissier, Leenhardt, Menegaux Chereau, Trésallet, Godiris Petit, Noullet, Tissier, Leenhardt, Menegaux

Chereau, Menegaux

Chereau, Trésallet, Menegaux

Chereau, Trésallet, Tissier, Leenhardt, Menegaux 


\section{ABSTRACT}

Background: Based on the AJCC 7th TNM classification, T1 intraglandular tumours are subdivided into T1a $(\leq 10 \mathrm{~mm})$ and T1b (11-20 mm), but the differences in prognosis remain controversial. The present study aimed to determine the clinicopathological features and outcomes of T1a and T1b patients.

Methods: A retrospective study of 2,518 T1 patients, including 1,840 T1a (73\%) and 678 (27\%) T1b patients who underwent surgery for PTC from 1978 to 2014, was conducted. In patients with a preoperative or operative diagnosis of PTC, a total thyroidectomy (TT) with prophylactic (macroscopically N0) or therapeutic (evident N1) lymph node dissection (LND) was performed. Other patients had a TT or partial thyroidectomy without LND. The mean follow-up time was $8.9 \pm 8.8$ years (median, 6.5 years; range, 1-36.4 years).

Results: A TT was performed in 2,273 patients (90\%), including 1,184 (52\%) with LND. Other patients (n=245) had a single lobectomy with isthmectomy. Multifocality, bilaterality, number of tumours, sum of the largest size of all foci, vascular invasion, and (in patients with LND) LN metastases were significantly more frequent in T1b than in T1a patients. Of the 1,184 patients with LND, 278 had LN metastases (N1, 23\%), including 136/680 T1a (20\%) and 142/504 (28\%) T1b patients ( $\mathrm{p}=0.002)$. These LN metastases were diagnosed after a prophylactic LND in 86/609 T1a (14\%) and 93/440 T1b (21\%) patients ( $\mathrm{p}=0.001)$. Recurrences were more frequent in T1b $(n=26,3.8 \%)$ than in T1a patients ( $\mathrm{n}=35$, $1.9 \%, \mathrm{p}=0.005)$. In the multivariate analysis, independent prognostic factors for recurrence in both groups were the number of tumours, the sum of the largest size of all foci and, in patients who had LND, LN metastases and extranodal extension. For N0-x patients, the recurrence rate was significantly higher in the T1b than in the T1a group (2.4\% vs. 0.9\%, respectively, $\mathrm{p}=0.005)$, although this rate was similar in $\mathrm{N} 1$ patients $(16.2 \%$ for $\mathrm{T} 1 \mathrm{a}$ and $9.2 \%$ for $\mathrm{T} 1 \mathrm{~b}$ patients, $\mathrm{p}=0.1)$. The 5-year disease-free survival rates for $\mathrm{T} 1 \mathrm{a}$ and $\mathrm{T} 1 \mathrm{~b}$ patients were $98.3 \%$ and $96.6 \%$, respectively $(\mathrm{p}=0.01)$. Conclusion: For PTC patients, T1b had poorer clinicopathological features and increased risk of recurrence than T1a.

\section{KEYWORDS}

Thyroid cancer, papillary; Recurrence; Lymph node dissection; Lymph node metastases; Thyroidectomy 


\section{INTRODUCTION}

Papillary thyroid cancers (PTCs) typically have an excellent prognosis, with an overall 10-year survival rate above 90\%. More than $80 \%$ of thyroid cancers are attributed to small PTCs (defined as tumours $\leq 20$ mm) [1]. Although most small PTCs have an indolent clinical course, several independent prognostic pejorative factors have been identified such as age $>45$ years, extrathyroidal extension, and lymph node (LN) and distant metastases. A large database study [2] concluded that tumours $>10 \mathrm{~mm}$ have a higher recurrence rate than do PTCs $\leq 10 \mathrm{~mm}$ and require more extensive thyroid surgery. Consequently, the 7th edition of the AJCC pTNM staging system [3] subdivided the T1 category of intraglandular tumours into T1a $(\leq 10 \mathrm{~mm})$ and T1b $(11-20 \mathrm{~mm})$, and the 2015 American Thyroid Association guidelines [4] recommended different therapeutic management for these 2 subgroups of PTC: a single lobectomy for T1a (unless there are clear indications to remove the contralateral lobe), a total thyroidectomy for T1b tumours, and post-surgical radioiodine therapy for T1bN0-x (N0 or Nx) with aggressive histology and for all T1 PTC with LN metastasis (N1). However, the prognostic value of a 10 -mm cutoff in maximum diameter remains controversial. Although it is well accepted that the recurrence rate closely correlates with a tumour size $>20 \mathrm{~mm}$ in PTC without preoperatively detected node metastasis [5], several studies have reported no difference in prognosis for small PTC tumours between 1 to 10 $\mathrm{mm}$ and 11 to $20 \mathrm{~mm}$ [6-8]. The aim of our study was to compare the clinicopathological features of T1a and T1b PTCs, excluding tumours with extrathyroidal extension, and to investigate the risk of recurrence of these two subgroups.

\section{PATIENTS AND METHODS}

A retrospective study of 2,518 consecutive patients who underwent surgery for T1 PTCs at our institution from January 1978 to December 2014 was conducted. Primary tumours $\leq 20$ mm with extrathyroidal extension (T3) were excluded from the study. Each patient underwent a systematic preoperative neck ultrasound (US). In patients with a preoperative 
(positive cytology) or operative (frozen section) diagnosis of thyroid carcinoma and a clinically negative nodal disease, we performed a total thyroidectomy with a prophylactic central (level VI) and lateral (levels III and IV, and level II if the cancer was located in the upper third of the thyroid lobe) lymph node dissection (LND) [9] on the side of the largest primary thyroid tumour. A therapeutic LND was performed in patients with clinically evident positive LN based on physical examination, neck US, or cytology. The remaining patients with a PTC diagnosed only on definitive pathology of thyroid resection had no LND (Nx). If PTC was identified by definitive pathology of a partial thyroid resection, complete thyroidectomy with ipsilateral neck dissection (central and lateral) was performed for T1b tumours only.

We collected patient demographic data (age, sex, and BMI). The following data were obtained from pathological examination: tumour size, number of tumours, sum of the largest size of all foci, multifocality and bilaterality, vascular invasion, number of resected LNs, number of LN metastases, and extranodal extension of positive LNs. During the postoperative course, indirect laryngoscopy was performed in patients with dyspnea, hoarseness, or loss of voice quality. When recurrent laryngeal nerve (RLN) palsy was diagnosed, a trial of speech therapy was started during the first week and continued over several months for a course of 30 treatments. RLN palsy was considered permanent if there was no proof of recovery using laryngoscopy within 6 months of operation. Serum calcium concentration was assessed on postoperative day 1 and repeated on postoperative day 2 if the first result was $<2 \mathrm{mmol} / \mathrm{L}$. Symptomatic hypocalcemia was defined as a serum calcium level $<2 \mathrm{mmol} / \mathrm{L}$ with overt manifestations, such as anxiety, carpopedal spasms, or tingling or numbness of the extremities. Hypoparathyroidism was considered permanent if the patient required oral calcium supplements and vitamin D for 6 months or more with a plasma parathyroid hormone level $<29.4 \mathrm{pmol} / \mathrm{L}$. Postoperative use of radioiodine ablation was also recorded. This treatment was administered for every T1b, every N1 patient, and any T1a with adverse features (vascular invasion; sum of the largest size of all foci $>10$ mm; or aggressive histologic subtypes, such as tall-cells, solid, columnar, oncocytic, or diffuse sclerosing variant). Radioiodine ablation was administered after withdrawing thyroid hormones for one month or (since 2010) with the use of recombinant human thyrotropin (r-TSH) in the lower-risk patient group. The radioactive ${ }^{131}$ I dose administered was 3.7 GBq systematically until August 2012. Beginning in September 2012, we administered 1.1 GBq under r-TSH for PTC patients with a low or intermediate risk of recurrence (T1a and T1b excluding aggressive histologic subtypes of PTC).

Follow-up information was obtained from a database of patient medical records. A standardized procedure was used for patient follow-ups, including check-ups under thyroid-stimulating hormone (TSH) stimulation at 6 months, 12 months, and annually thereafter. After 7 years of follow-up, periodic correspondence with patients or their referring physicians was designed to occur every 3 years. Physical examinations, neck US, and serum thyroglobulin (Tg) measurements after stimulation or under suppressive treatment were routinely performed during the follow-ups. 
Events were analyzed retrospectively. Events that were considered recurrent included the following: locoregional recurrence defined as a thyroid bed, soft tissue, or cervical lymph node recurrence of PTC. The diagnosis of locoregional recurrence was assessed by histological analysis at surgery, by fine-needle aspiration biopsy or by 131I cervical uptake anywhere in the neck after a 131I diagnostic (11.1 MBq) or therapeutic (3.7 GBq) dose. In addition, patients with isolated and repeatedly elevated serum thyroglobulin levels ( $>10 \mathrm{ng} / \mathrm{ml}$ after T4 withdrawal) that justified the administration of a second therapeutic dose of 131I and patients with distant metastases assessed by cytological or histological analysis or by iodine uptake after diagnostic or therapeutic scintigraphy were also considered to have recurrent disease.

\section{Statistical Analysis}

Data analysis was performed using SPSS (version 21, IBM Corporation, Armonk, NY). Statistical analysis of the differences between clinical and histological variables between groups was conducted by chi-2 tests or Fisher's exact test as appropriate. The Mann-Whitney U test was used to compare continuous variables, as we could not assume a normal distribution because of the relatively small numbers. A p value less than 0.05 was regarded as significant. A univariate logistic regression was used to estimate the relationship between recurrence and the following variables: patient sex, age, BMI, number of tumours (multifocality, bilaterality), sum of the largest foci sizes, vascular invasion, and LN metastases. We performed a Cox regression multivariate analysis that included the predictive factors that had a significant relationship with recurrence in the univariate analysis; odds ratios (ORs) were estimated by the use of an unconditional logistic regression model. Disease-free survival was calculated using the Kaplan-Meier method and log-rank test.

\section{RESULTS}

\section{Characteristics of the study cohort}

During the study period, 2,518 patients underwent surgery for T1 PTCs, including 1,840 (73\%) T1a and 678 T1b tumours. Indication for surgery was a preoperative diagnosis of PTC in 547 patients (22\%) (positive ultrasound-guided fine-needle aspiration cytology). An additional group of 637 patients (25\%) had a positive frozen section during surgery and received, like the previous group, a total thyroidectomy with LND. For the remaining patients (n=1,334; 53\%), PTC was diagnosed only on final pathology of a thyroid specimen, including 1,160 T1a (63\% of all T1a patients) and 174 T1b (26\%) $(\mathrm{p}<0.0001)$ (Table 1). Of the entire cohort, 2,273 patients had a total thyroidectomy, including 1,184 with LND. Other patients ( $\mathrm{n}=245)$ had a single lobectomy with isthmectomy.

The results of recurrent laryngeal nerve palsies and postoperative hypoparathyroidism are summarized in Table 1 . The permanent complications rate was 3.2\% in T1a patients $(n=58)$ and 3.7\% in T1b patients (n=25). We found no significant difference in the rate of permanent complications between patients with or without lymph node dissection (LND): 
22/680 LND (3.2\%) vs. 36/1160 Nx (3.1\%) in the T1a group (p=0.9) and 19/504 LND (3.8\%) vs. 6/174 Nx (3.4\%) in the T1b group ( $\mathrm{p}=0.8)$.

The pathological features of the T1 PTC patients are shown in Table 2. The number of tumours (2.3 vs. 1.6) and the sum of the largest size of all foci $(19$ vs. $6 \mathrm{~mm})$ were greater in T1b than in T1a patients $(\mathrm{p}<0.0001)$, and vascular invasion ( $8 \%$ vs. $1 \%)$, multifocality ( $45 \%$ vs. $29 \%$ ), and bilaterality ( $27 \%$ vs. $14 \%$ ) of the tumour were significantly more frequent in T1b than in T1a patients ( $\mathrm{p}<0.0001)$. T1b patients had a higher rate of LND than did T1a patients (74\% vs. $37 \%, \mathrm{p}<0.0001)$. In patients who had a LND (1,184 patients), metastases to the lymph nodes (N1) were more frequent in T1b than in T1a patients (142/504 patients, 28\%, vs. 136/680 patients, 20\%, respectively, $\mathrm{p}=0.002)$, and the number of involved LNs was greater in T1b than in T1a patients (median, 3 [1-23] vs. 1 [1-23], respectively, p=0.001). Central and lateral LN metastases were significantly more frequent in the T1b group: 123 (24\%) and 73 (14\%), respectively, in T1b patients, 106 (16\%) and 57 (8\%), respectively, in T1a patients ( $\mathrm{p}=0.0001)$. Of the $130 \mathrm{~N} 1 \mathrm{~b}$ patients (57 T1a and 73 T1b), 49 (38\%) had isolated lateral LN metastasis (skip metastasis) (30 T1a and $19 \mathrm{~T} 1 \mathrm{~b}, \mathrm{p}=0.001$ ).

Of the patients with a prophylactic LND (n=1,049), 179 had LN metastases (17\%), including 86/609 T1a (14\%) patients and 93/440 T1b (21\%) $(\mathrm{p}=0.001)$. The lateral compartment (N1b) was involved in 61 (34\%) of these patients, including 22/86 T1a (26\%) and 39/93 T1b (42\%) patients ( $\mathrm{p}=0.02)$

A total of 1,248 patients (50\%) received radioiodine therapy, including 603 (33\%) T1a and 645 (95\%) T1b patients.

There was no difference between T1a and T1b patients in term of ${ }^{131} \mathrm{I}$ dose (mean, $3.7 \pm 0.3 \mathrm{GBq}$ vs. $3.5 \pm 0.4 \mathrm{GBq}$, respectively).

\section{Follow-up and oncologic outcomes}

Postoperative follow-up ranged from 2 to 36.4 (median, 6.5) years. Recurrence was observed in 61 patients (2.4\%), including $35 \mathrm{~T} 1 \mathrm{a}(1.9 \%)$ and $26 \mathrm{~T} 1 \mathrm{~b}$ patients $(3.8 \%)(\mathrm{p}=0.005)$ (Table 3). The median time from initial surgery to recurrence was similar in both groups: 1.6 years (range, 0.2-8.9 years) for T1b patients, and 1.1 years (range, 0.2-12 years) for T1a patients ( $\mathrm{p}=0.5$ ). Locoregional recurrence occurred in 45 patients ( $74 \%$ of recurrences), all of whom underwent reoperative surgery. Other patients had distant metastases $(n=4)$ or elevated isolated serum thyroglobulin levels $(n=12)$ and were exclusively treated with repeated iodotherapy. Five patients (4 T1a and $1 \mathrm{~T} 1 \mathrm{~b})$ died from distant metastases of PTC.

Of the N1 patients $(n=278)$, the recurrence rates were equivalent between T1a and T1b patients, with rates of $16.2 \%$ (22/136) and a 9.2\% (13/142), respectively $(\mathrm{p}=0.1)$. These recurrence rates were higher than those found in N0 and Nx patients: $0.7 \%$ (4/544) of T1a N0 (p<0.001), 0.8\% (9/1,160) of T1a Nx ( $<<0.001), 1.4 \%(5 / 362)$ of T1b N0 ( $<0.001)$ and $4.6 \%(8 / 174)$ of T1b Nx patients $(\mathrm{p}=0.1)$. 
When the T1a N0-x and T1bN0-x patient subgroups were compared, we found a higher recurrence rate in the T1b N0-x group: $2.4 \%(13 / 537)$ in the T1bN0-x vs. $0.9 \%$ in the T1a N0-x group $(16 / 1703)(\mathrm{p}=0.005)$. In patients who had no LND (Nx), T1b patients had a higher risk of recurrence than T1bN0 patients (4.6\% vs. 1.4\%, respectively; $\mathrm{p}=0.02)$, whereas no difference was observed in T1a patients ( $0.8 \%$ for T1aNx vs. $0.7 \%$ for T1aN0; $\mathrm{p}=0.8)$.

Within the groups of $\mathrm{T} 1 \mathrm{a}$ and $\mathrm{T} 1 \mathrm{~b}$ patients, the univariate analysis revealed a significantly higher risk of recurrence relative to tumour size, multifocality, including number of tumours, sum of the largest size of all foci, lymph node metastases and extranodal extension (Table 4). Age >45years, vascular invasion and distant metastases in T1a patients and male gender and bilaterality in T1b patients were also significantly associated with recurrence. In the multivariate analysis, the number of tumours (OR=1.7; 95\%CI [1.3-2.1] for T1a and OR=1.5; 95\%CI [1.2-2.6] for T1b), the sum of the largest size of all foci (OR=2.7; 95\%CI [2-3.6] and OR=3.2; 95\%CI [2.3-4.5]), and in patients who had LND, lymph node metastases (OR=9.5; 95\%CI [4.7-14.2] for T1a and OR=4.1; 95\%CI [1.2-9.4] for T1b) and extranodal extension (OR=12.2; 95\%CI [2.3-31] and $\mathrm{OR}=4.2$; 95\%CI [2-6.4]) remained independent risks factors for recurrence in both T1a and T1b groups.

The 5-year disease-free survival (DFS) was high but significantly different between the T1a and T1b patients (98.3\% vs. 96.6\%, respectively; $\mathrm{p}=0.01$ ). A log-rank test showed that the DFS rate was significantly greater in the T1a than in the T1b group $(\mathrm{p}=0.01)$ (figure 1$)$.

\section{DISCUSSION}

It is still unclear in the current literature whether T1a and T1b patients have similar prognoses and whether the therapeutic management of these patients should differ. In the present study, we report that the clinicopathological features and risk of recurrence are not similar between T1a and T1b patients, with a higher incidence of multifocality including the number of tumours, bilaterality, vascular invasion and lymph node metastases in T1b patients and a poorer prognosis with an increased risk of recurrence and a lower DFS for T1b patients with a tumour $>10 \mathrm{~mm}$.

We found a higher rate of incidentally diagnosed PTCs in T1a than in T1b patients (63\% vs. 26\%, p<0001). Because we performed a systematic LND in case of preoperative or operative diagnosis of PTC, LND was significantly more frequent in T1b than in T1a patients in our study. For patients with LND, T1b PTC had a significantly higher rate of LN metastases than did T1a PTC (28\% vs. 20\%, p=0.002). This finding is in agreement with the importance of tumour size, even in small PTC, as a strong predictive factor of LN metastases [10]. Reddy et al. [11] reported no difference in LN spread between tumours less than $1 \mathrm{~cm}$ and those between 1 and $2 \mathrm{~cm}$; however, they only evaluated patients who were referred for radioiodine therapy, with an unusually high rate of $\mathrm{LN}$ metastases (45\%) for tumours $<2 \mathrm{~cm}$. 
Several authors have already reported different clinicopathological features and outcomes for tumours $\leq 10 \mathrm{~mm}$ and those 11-20 mm, but they have usually included small tumours with extrathyroidal extension (ETE) and heterogeneous thyroid carcinoma including follicular cancers [6, 10-14]. However, it is well-recognized that one of the most pejorative factors in the risk of PTC recurrence is the presence of ETE [10]. For example, Ito et al [8] investigated the prognosis of 2,638 patients with PTCs measuring $20 \mathrm{~mm}$ or less who did not have a massive ETE (T4), clinically apparent lymph nodes or distant metastasis. They reported that the disease-free survival (DFS) was not affected by tumour size ( $\leq 10$ mm vs. 11-20 mm); however, 21\% of their PTCs had a minimal ETE of the tumour (T3). Wang et al [7] conducted the first study that was limited to small PTCs without ETE (T1a and T1b). They reported similar rates of recurrence in T1a and T1b patients (1.2\%) with a 5-year DFS that was identical for both groups $(98.6 \%, \mathrm{p}=0.2)$. However, the median follow-up was relatively short in this study (46 months), and the standard postoperative check-up procedure was not described. With a longer follow-up, we reported a 2-fold risk of recurrence in T1b patients when compared to T1a patients (3.8\% vs. $1.9 \%$, respectively, $\mathrm{p}=0.005)$. We therefore believe that these two categories of small PTC do not have similar outcomes and that the subdivision of the T1 category into T1a and T1b (AJCC staging 7th edition) seems to be justified.

A prophylactic LND remains controversial for T1 PTCs. Although it is useful in determining accurate PTC staging by estimating the risk stratification and by facilitating management of the disease, follow-up strategies and postsurgical administration of $131 \mathrm{I}[15,16]$, it is not recommended in the current guidelines $[4,17,18]$ for clinically node-negative small PTCs. The rationale behind these guidelines is the lack of proven benefit of its long-term clinical outcome and the risk of surgical complications associated with central lymph node dissection, i.e., recurrent laryngeal nerve palsy and hypoparathyroidism. However, the present study demonstrated that our strategy of performing systematic prophylactic lymph node dissection was not responsible for a significant increase in the rate of postoperative complications. Moreover, prophylactic central and lateral LNDs revealed LN metastases in 17\% of T1 patients, including 21\% of T1b and 14\% of T1a patients. Machens et al. [14] analyzed the correlation between primary tumour size and risk of extrathyroidal extension, lymph node and distant metastasis in 366 PTC and 134 follicular thyroid carcinomas (FTC). The cumulative risks increased linearly with increasing tumour diameter, even in small PTC $<20 \mathrm{~mm}$. They reported that the smallest size for developing extrathyroidal extension and lymph node metastases was 5 mm for PTC. They also showed that the cumulative risk of distant metastasis increased once the primary tumour size was 20 mm. However, in our study including 960 PTC with a primary tumour size less than 5 mm, 14.4\% of patients with LND had lymph node metastases (28/194). Furthermore, distant metastases were present in $11 \mathrm{~T} 1$ patients including 9 T1a patients. Consequently, we recommend a surgical strategy with systematic prophylactic lymph node dissection for all T1 PTC tumours, including for tumour sizes $<5 \mathrm{~mm}$. 
Among the 1,248 patients who had a radioiodine ablation in our institution (including 645 T1b and 603 T1a patients), 93 T1b (14\%) and 86 T1a patients (14\%) had LN metastasis discovered after a prophylactic LND. Moreover, if the prophylactic LND had been limited to the central compartment, 61 patients with lateral LN metastases (N1b) would not have been detected. We also found that the prognosis of T1b patients who did not undergo LND (Nx) was significantly poorer than it was for N0 patients and that LN metastases (N1) were also associated with a higher risk of recurrence than N0-x in the 2 subgroups of T1a and T1b patients. In the multivariate analysis, LN metastases and extranodal extension were found to contribute to the risk of recurrence in T1a and T1b patients. However, although these findings emphasize the utility of performing prophylactic central and lateral LND to assess an accurate staging of small PTC, we also report that it did not improve the prognosis of $\mathrm{T} 1$ patients and that similar results have been obtained in previous studies [8, 19].

Although we analyzed a large cohort of consecutive T1 patients, our study has several limitations, including its retrospective nature. However, we believe that our homogeneous management of PTC patients, including surgical procedures with systematic prophylactic LND during the study period, overcomes the potential limitations of the study.

In conclusion, T1b patients have poorer clinicopathological features and increased risk of recurrence than T1a patients. A systematic prophylactic LND should be considered even in these small tumours to guide the indication for radioiodine therapy and to determine the intervals of post-surgical follow up. 


\section{REFERENCES}

1. Davies L, Welch HG. Increasing incidence of thyroid cancer in the United States, 1973-2002. JAMA. 2006 May $10 ; 295(18): 2164-7$.

2. Bilimoria KY, Bentrem DJ, Linn JG, Freel A, Yeh JJ, Stewart AK, Winchester DP, Ko CY, Talamonti MS, Sturgeon

C. Utilization of total thyroidectomy for papillary thyroid cancer in the United States. Surgery. 2007 Dec;142(6):90613.

3. Edge SB, Compton CC. The American Joint Committee on Cancer: the 7th edition of the AJCC cancer staging manual and the future of TNM. Ann Surg Oncol. 2010 Jun;17(6):1471-4.

4. American Thyroid Association Management Guidelines for Adult Patients with Thyroid Nodules and Differentiated Thyroid Cancer. Thyroid 2015

5. Ito Y, Fukushima M, Higashiyama T, Kihara M, Takamura Y, Kobayashi K, Miya A, Miyauchi A. Tumor size is the strongest predictor of microscopic lymph node metastasis and lymph node recurrence of N0 papillary thyroid carcinoma. Endocr J. 2013;60(1):113-7.

6. Pellegriti G, Scollo C, Lumera G, Regalbuto C, Vigneri R, Belfiore A. Clinical behavior and outcome of papillary thyroid cancers smaller than 1.5 cm in diameter: study of 299 cases. J Clin Endocrinol Metab. 2004 Aug;89(8):3713-20. 7. Wang LY, Nixon IJ, Palmer FL, Thomas D, Tuttle RM, Shaha AR, Patel SG, Shah JP, Ganly I. Comparable outcomes for patients with pT1a and pT1b differentiated thyroid cancer: Is there a need for change in the AJCC classification system? Surgery. 2014 Dec;156(6):1484-9; discussion 1489-90.

8. Ito Y, Masuoka H, Fukushima M, Inoue H, Kihara M, Tomoda C, Higashiyama T, Takamura Y, Kobayashi K, Miya A, Miyauchi A. Excellent prognosis of patients with solitary T1N0M0 papillary thyroid carcinoma who underwent thyroidectomy and elective lymph node dissection without radioiodine therapy. World J Surg. 2010 Jun;34(6):1285-90. 9. Ducoudray R, Trésallet C, Godiris-Petit G, Tissier F, Leenhardt L, Menegaux F. Prophylactic lymph node dissection in papillary thyroid carcinoma: is there a place for lateral neck dissection? World J Surg 2013;37:1584-91.

10. Ito Y, Miyauchi A, Kihara M, Kobayashi K, Miya A. Prognostic values of clinical lymph node metastasis and macroscopic extrathyroid extension in papillary thyroid carcinoma. Endocr J. 2014;61(8):745-50.

11. Reddy RM, Grigsby PW, Moley JF, Hall BL. Lymph node metastases in differentiated thyroid cancer under 2 cm. Surgery. 2006 Dec;140(6):1050-4; discussion 1054-5. 
12. Rossi R, Roti E, Trasforini G, Pansini G, Cavazzini L, Zatelli MC, Pearce EN, Braverman LE, Uberti EC. Differentiated thyroid cancers 11-20 mm in diameter have clinical and histopathologic characteristics suggesting higher aggressiveness than those $<$ or =10 mm. Thyroid. 2008 Mar;18(3):309-15.

13. Nilubol N, Kebebew E. Reply to most patients with a small papillary thyroid carcinoma enjoy an excellent prognosis and may be managed with minimally invasive therapy or active surveillance. Cancer. 2015 Sep 15;121(18):3365-6.

14. Machens A, Holzhausen HJ, Dralle H The prognostic value of primary tumor size in papillary and follicular thyroid carcinoma. Cancer 2005; 103:2269-2273.

15. Bonnet S, Hartl D, Leboulleux S, Baudin E, Lumbroso JD, Al Ghuzlan A, Chami L, Schlumberger M, Travagli JP. Prophylactic lymph node dissection for papillary thyroid cancer less than $2 \mathrm{~cm}$ : implications for radioiodine treatment. $\mathrm{J}$ Clin Endocrinol Metab. 2009 Apr;94(4):1162-7.

16. Hartl DM, Leboulleux S, Al GA, Baudin E, Chami L, Schlumberger M, Travagli JP. Optimization of staging of the neck with prophylactic central and lateral neck dissection for papillary thyroid carcinoma. Ann Surg 2012; 255:777-783 17. Sancho JJ, Lennard TW, Paunovic I, Triponez F, Sitges-Serra A Prophylactic central neck disection in papillary thyroid cancer: a consensus report of the European Society of Endocrine Surgeons (ESES). Langenbecks Arch Surg 2014; 399:155-163

18. Dralle H et al., German Societies of General and Visceral Surgery; Endocrinology; Nuclear Medicine; Pathology; Radiooncology; Oncological Hematology; and the German Thyroid Cancer Patient Support Organization Ohne Schilddrüse leben e.V. German Association of Endocrine Surgeons practice guideline for the surgical management of malignant thyroid tumors. Langenbecks Arch Surg. 2013 Mar;398(3):347-75.

19. Qu N, Zhang L, Ji QH, Chen JY, Zhu XY, Cao YM, Shen Q. Risks factors for central lymph node metastasis in papillary thyroid microcarcinoma: a meta-analysis. World J Surg 2015; 39(10):2459-70 


\begin{tabular}{|c|c|c|c|}
\hline Factor & T1a $(n=1,840)$ & T1b (n=678) & $\mathrm{p}$ value \\
\hline Sex, Female & $1,515(82)$ & $544(80)$ & 0.3 \\
\hline Age, years & $52(15-88)$ & $46(11-85)$ & $<0.0001$ \\
\hline BMI (kg/m²) & $25(16-56)$ & $24(17-46)$ & 0.02 \\
\hline \multicolumn{4}{|l|}{ Indications for Thyroid Surgery } \\
\hline Preoperative diagnosis of PTC by FNAB & $369(20)$ & $178(26)$ & 0.001 \\
\hline Positive frozen section during surgery & $311(17)$ & $326(48)$ & $<0.0001$ \\
\hline Incidental carcinomas & $1,160(63)$ & $174(26)$ & $<0.0001$ \\
\hline Euthyroid multinodular goiter & 746 & 120 & \\
\hline Hyperthyroidism: & 256 & 33 & \\
\hline _ Grave’s disease & 99 & 7 & \\
\hline _ Toxic multinodular goiter & 137 & 17 & \\
\hline _ Toxic adenoma & 20 & 9 & \\
\hline Suspicious nodule with negative frozen section & 158 & 21 & \\
\hline \multicolumn{4}{|l|}{ Thyroid surgery } \\
\hline Total thyroidectomy & $1,668(91)$ & $605(89)$ & 0.3 \\
\hline One step & $1,451(87)$ & $507(84)$ & \\
\hline Two steps & $217(13)$ & $98(16)$ & \\
\hline Lobectomy & $172(9)$ & $73(11)$ & 0.3 \\
\hline LND & $680(37)$ & $504(74)$ & $<0.0001$ \\
\hline Therapeutic & $71(10)$ & $64(13)$ & 0.2 \\
\hline Prophylactic & $609(90)$ & $440(87)$ & 0.2 \\
\hline No LND (Nx) & $1,160(63)$ & $174(26)$ & \\
\hline \multicolumn{4}{|l|}{ Surgical Complications } \\
\hline Hypoparathyroidism & $268(14.6)$ & 128 (18.9) & 0.008 \\
\hline Permanent & 41 & 21 & \\
\hline Transient & 227 & 107 & \\
\hline Recurrent laryngeal nerve paralysis & $47(2.6)$ & $26(3.8)$ & 0.09 \\
\hline Permanent & 17 & 4 & \\
\hline Transient & 30 & 22 & \\
\hline Radioiodine Therapy & $603(33)$ & $645(95)$ & $<0.001$ \\
\hline Mean Dose, GBq & $3.7 \pm 0.3$ & $3.5 \pm 0.4$ & 0.5 \\
\hline
\end{tabular}

TABLE 1. Clinical and surgical characteristics of the T1 PTC patients $(n=2,518)$ 
TABLE 2. Pathological features of T1 PTC patients $(n=2,518)$

\begin{tabular}{|c|c|c|c|}
\hline Factor & $\begin{array}{c}\mathrm{T} 1 \mathrm{a} \\
(\mathrm{n}=1,840)\end{array}$ & $\begin{array}{c}\text { T1b } \\
(n=678)\end{array}$ & $\mathrm{p}$ value \\
\hline \multicolumn{4}{|l|}{ Primary tumor } \\
\hline Size, mm & $4(1-10)$ & $15(11-20)$ & \\
\hline Multifocality & 535 (29) & $305(45)$ & $<0.0001$ \\
\hline Bilaterality & $256(14)$ & $182(27)$ & $<0.0001$ \\
\hline Number of tumors & $1.6 \pm 0.9$ & $2.3 \pm 1.5$ & $<0.0001$ \\
\hline Sum of the largest size of all foci (mm) & $5.9 \pm 0.7$ & $18.7 \pm 4.8$ & $<0.0001$ \\
\hline Vascular invasion & $19(1)$ & $52(8)$ & $<0.0001$ \\
\hline Results of $L N D(n=1,184)$ & $680(37)$ & $504(74)$ & $<0.0001$ \\
\hline Number of excised LN & $14(14.1-76)$ & $17(1-72)$ & 0.0003 \\
\hline LN metastasis (N1) & $136(20)$ & $142(28)$ & 0.002 \\
\hline Number of involved LN & $1(1-23)$ & $3(1-23)$ & 0.001 \\
\hline Extranodal extension & $22(16)$ & $25(18)$ & 0.7 \\
\hline Distant Metastases (M1) & $9(0.5)$ & $2(0.1)$ & 0.8 \\
\hline
\end{tabular}

Data are presented as $\mathrm{n}(\%)$, mean \pm standard deviation or as median (range). LND: lymph node dissection 
TABLE 3. Oncologic outcomes of the studied T1 PTC patients $(n=2,518)$

\begin{tabular}{|c|c|c|c|}
\hline Variable & $\begin{array}{c}\mathrm{T} 1 \mathrm{a} \\
(\mathrm{n}=1,840)\end{array}$ & $\begin{array}{c}\text { T1b } \\
(n=678)\end{array}$ & $\mathrm{p}$ \\
\hline Global recurrence*, n (\%) & $35(1.9)$ & $26(3.8)$ & 0.005 \\
\hline 1/ No LND (Nx) and N0 & $13 / 1,704(0.8)$ & $13 / 536(2.4)$ & $<0.001$ \\
\hline $\mathrm{Nx}$ & $9 / 1,160(0.8)$ & $8 / 174(4.6)$ & $<0.001$ \\
\hline N0 & $4 / 544(0.7)$ & 5/362 (1.4) & 0.3 \\
\hline 2/ N1 & 22/136 (16.2) & 13/142 (9.2) & 0.1 \\
\hline Locoregional recurrence, n (\%) & $23(1.3)$ & $22(3.2)$ & $<0.001$ \\
\hline 1/ No LND (Nx) and N0 & $9(0.5)$ & $11(2.1)$ & 0.001 \\
\hline Nx & $6(0.5)$ & $8(4.6)$ & $<0.001$ \\
\hline N0 & $3(0.6)$ & $3(3.8)$ & 0.6 \\
\hline 2/ N1 & $14(10.3)$ & $11(7.7)$ & 0.5 \\
\hline Time to recurrence, (median, range) (years) & $1.1(0.2-12)$ & $1.6(0.2-8.9)$ & 0.5 \\
\hline Disease-specific mortality, n (\%) & $4(0.2)$ & $1(0.1)$ & 0.7 \\
\hline
\end{tabular}

* : includes locoregional recurrence, distant metastasis, or isolated elevated serum thyroglobulin levels 


\begin{tabular}{|c|c|c|c|c|c|c|c|c|}
\hline \multirow[b]{2}{*}{ Variable } & \multicolumn{4}{|c|}{ T1a ( $\mathrm{n}=1,840$ patients) } & \multicolumn{4}{|c|}{ T1b (n=678 patients) } \\
\hline & $\begin{array}{l}\text { Event } \\
(\mathrm{n}=35)\end{array}$ & $\begin{array}{l}\text { No event } \\
(n=1,805)\end{array}$ & $\begin{array}{c}\text { Univariate } \\
\text { analysis } \\
\text { p }\end{array}$ & $\begin{array}{c}\text { Multivariate } \\
\text { Analysis } \\
\text { OR }(95 \% \mathrm{CI})\end{array}$ & $\begin{array}{c}\text { Event } \\
(\mathrm{n}=26)\end{array}$ & $\begin{array}{l}\text { No event } \\
(\mathrm{n}=652)\end{array}$ & $\begin{array}{c}\text { Univariate } \\
\text { analysis } \\
\text { p }\end{array}$ & $\begin{array}{l}\text { Multivariate } \\
\text { Analysis } \\
\text { OR }(95 \% \mathrm{CI})\end{array}$ \\
\hline Sex Male & $3(9)$ & $322(18)$ & 0.2 & & $10(38)$ & $124(18)$ & 0.01 & $2.1(0.4-16.2)$ \\
\hline Age $>45$ years & $17(49)$ & $1193(66)$ & 0.03 & & $13(50)$ & 348 (53) & 0.7 & \\
\hline BMI, kg/m² & $22.7+-2.8$ & $25.4+-3.9$ & 0.1 & & $25.3+-4.4$ & $24.8+-3.6$ & 0.3 & \\
\hline Size, mm & $5.8+-2.7$ & $4.6+-2.6$ & 0.002 & $1.8(0.5-7.9)$ & $14.7+-2.3$ & $15.3+-2.6$ & $<0.0001$ & \\
\hline Multifocality & $16(46)$ & 519 (29) & 0.03 & $1.5(0.3-6.2)$ & $17(65)$ & $288(44)$ & 0.03 & $1.9(0.4-5.2)$ \\
\hline Bilaterality & $7(20)$ & 249 (14) & 0.3 & & $14(54)$ & $168(26)$ & 0.001 & $2.3(0.7-7.3)$ \\
\hline Number of tumors & $2.5+-1.8$ & $1.6+-0.8$ & 0.001 & $1.7(1.3-2.1)$ & $3+-1.9$ & $2.2+-1.5$ & 0.01 & $1.5(1.2-2.6)$ \\
\hline $\begin{array}{l}\text { Sum of the largest size of } \\
\text { all foci, mm }\end{array}$ & $8+-5$ & $5.9+-3.7$ & $<0.0001$ & $2.7(2-3.6)$ & $21.3+-5.9$ & $18.6+-4.7$ & $<0.0001$ & $3.2(2.3-4.5)$ \\
\hline Vascular invasion & $2(6)$ & $17(1)$ & 0.006 & $1.2(0.4-1.8)$ & 0 & $52(8)$ & 0.1 & \\
\hline LND & $26(74)$ & $654(36)$ & $<0.0001$ & & $18(69)$ & $486(75)$ & 0.5 & \\
\hline Lymph node metastases & $22(63)$ & $116(6)$ & $<0.0001$ & $9.5(4.7-14.2)$ & $13(50)$ & $129(20)$ & 0.0002 & $4.1(1.2-9.4)$ \\
\hline N1b & 16 & 41 & $<0.0001$ & & 10 & 63 & $<0.0001$ & \\
\hline N1a & 6 & 73 & 0.0002 & & 3 & 66 & 0.8 & \\
\hline Extranodal extension & $4(11)$ & $18(1)$ & $<0.0001$ & $12.2(2.3-31)$ & $3(12)$ & $22(3)$ & 0.03 & $4.2(2-6.4)$ \\
\hline Metastases & $3(9)$ & $6(0.3)$ & $<0.0001$ & $10(6.7-28.4)$ & 0 & $2(0.3)$ & 0.7 & \\
\hline Radioiodine therapy & $31(53)$ & $572(32)$ & $<0.0001$ & & $21(81)$ & $624(96)$ & $<0.001$ & \\
\hline
\end{tabular}

Data are presented as $\mathrm{n}(\%)$ or as the mean \pm standard deviation; LND: Lymph node dissection. Table 4. Univariate and multivariate analysis to identify prognosis factors for recurrence in T1a and T1b patients related to clinicopathological characteristics. 


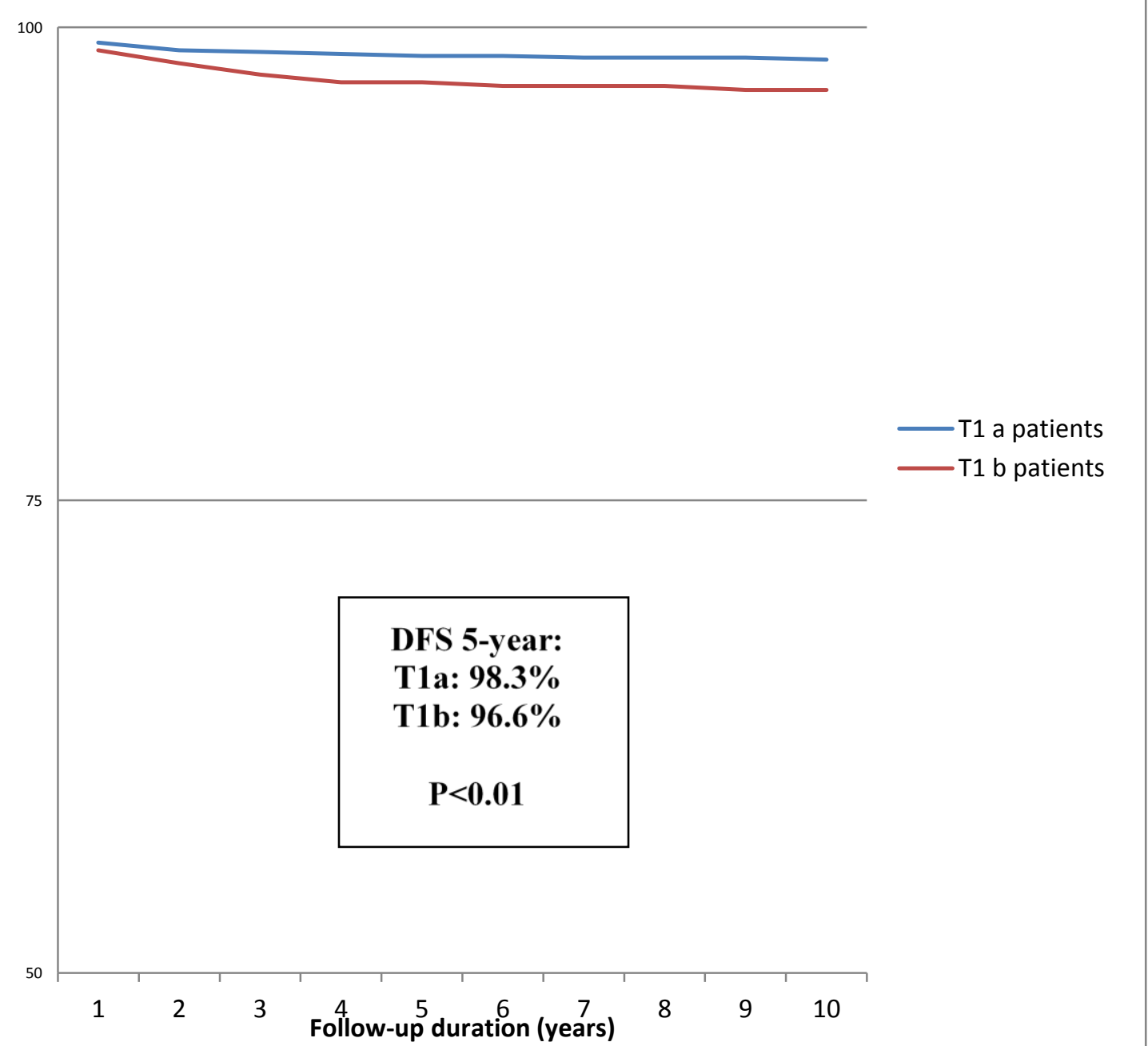

Figure 1. Disease-free survival (DFS) curves of the T1a $(n=1,840)$ and T1b $(n=678)$ patients 